\title{
Prediction of Motor Recovery Using Quantitative Parameters of Motor Evoked Potential in Patients With Stroke
}

\author{
Jae Yong Jo, $\mathrm{MD}^{1}$, Ahee Lee, $\mathrm{MS}^{2}$, Min Su Kim, $\mathrm{MD}^{3}$, Eunhee Park, $\mathrm{MD}^{1}$, \\ Won Hyuk Chang, MD, $\mathrm{PhD}^{1}$, Yong-Il Shin, MD, $\mathrm{PhD}^{4}$, Yun-Hee Kim, MD, $\mathrm{PhD}^{1,2}$
}

\begin{abstract}
${ }^{1}$ Department of Physical and Rehabilitation Medicine, Center for Prevention and Rehabilitation, Heart Vascular and Stroke Institute, Samsung Medical Center, Sungkyunkwan University School of Medicine, Seoul; ${ }^{2}$ Department of Health Science and Technology, Department of Medical Device Management \& Research, SAIHST, Sungkyunkwan University, Seoul; ${ }^{3}$ Department of Rehabilitation Medicine, Wonkwang University School of Medicine, Iksan; ${ }^{4}$ Department of Rehabilitation Medicine, Pusan National University School of Medicine \& Research Institute for Convergence of Biomedical Science and Technology, Pusan National University Yangsan Hospital, Pusan National University School of Medicine, Yangsan, Korea
\end{abstract}

Objective To investigate the clinical significance of quantitative parameters in transcranial magnetic stimulation (TMS)-induced motor evoked potentials (MEP) which can be adopted to predict functional recovery of the upper limb in stroke patients in the early subacute phase.

Methods One hundred thirteen patients ( 61 men, 52 women; mean age $57.8 \pm 12.2$ years) who suffered faiarst-ever stroke were included in this study. All participants underwent TMS-induced MEP session to assess the corticospinal excitability of both hand motor cortices within 3 weeks after stroke onset. After the resting motor threshold (rMT) was assessed, five sweeps of MEP were performed, and the mean amplitude of the MEP was measured. Latency of MEP, volume of the MEP output curve, recruitment ratios, and intracortical inhibition and facilitation were also measured. Motor function was assessed using the Fugl-Meyer Assessment scale (FMA) within 3 weeks and at 3 months after stroke onset. Correlation analysis was performed between TMS-induced MEP derived measures and FMA scores.

Results In the MEP response group, rMT and rMT ratio measures within 3 weeks after stroke onset showed a significant negative correlation with the total and upper limb FMA scores at 3 months after stroke $(p<0.001)$. Multiple regression analysis revealed that FMA score and rMT ratio, but not rMT within 3 weeks were independent prognostic factors for FMA scores at 3 months after stroke.

Conclusion These results indicated that the quantitative parameter of TMS-induced MEP, especially rMT ratio in the early subacute phase, could be used as a parameter to predict motor function in patients with stroke.

Keywords Motor recovery, Motor evoked potentials (MEP), Transcranial magnetic stimulation, Stroke, Prognosis

Received December 30, 2015; Accepted March 23, 2016

Corresponding author: Yun-Hee Kim

Department of Physical and Rehabilitation Medicine, Center for Prevention and Rehabilitation, Heart Vascular and Stroke Institute, Samsung Medical Center, Sungkyunkwan University School of Medicine, 81 Irwon-ro, Gangnam-gu, Seoul 06351, Korea. Tel: +82-2-3410-2824, 2818, Fax: +82-2-34100388, E-mail: yunkim@skku.edu, yun1225.kim@samsung.com

ORCID: Jae Yong Jo (http://orcid.org/0000-0002-0878-6779); Ahee Lee (http://orcid.org/0000-0002-8150-2170); Min Su Kim (http://orcid.org/00000001-9954-1445); Eunhee Park (http://orcid.org/0000-0001-6553-2321); Won Hyuk Chang (http://orcid.org/0000-0002-4969-7895); Yong-Il Shin (http://orcid.org/0000-0001-7894-0930); Yun-Hee Kim (http://orcid.org/0000-0001-6101-8851).

@ This is an open-access article distributed under the terms of the Creative Commons Attribution Non-Commercial License (http://creativecommons.org/ licenses/by-nc/4.0) which permits unrestricted noncommercial use, distribution, and reproduction in any medium, provided the original work is properly cited. Copyright $\odot 2016$ by Korean Academy of Rehabilitation Medicine 


\section{INTRODUCTION}

Stroke is one of the most important issues encountered by the world's aging population and it is a leading cause of long-term disability [1]. Among many symptoms of stroke, motor recovery may be the most important aspect in stroke patients for performing functionally independent activities of daily life. Actually, it has been reported that motor impairment is one of the most serious disabling sequelae of stroke, with more than $50 \%$ of stroke patients experiencing a residual motor deficit [2]. To establish a proper rehabilitation plan for motor impairment, many studies were conducted to predict outcomes of motor function using various tools. Lesion localization and functional reorganization revealed possible predictive tools using various imaging studies [3-5]. Usefulness of diffusion tensor tractography to predict motor recovery has also been investigated in many studies [6]. Furthermore, transcranial magnetic stimulation (TMS)-induced motor evoked potentials (MEP) performed during hospital admission can be the method to predict functional recovery [7-10]. In general, responsiveness of MEP is an important prognostic factor to predict motor recovery in stroke patients [7-14].

TMS-induced MEP can provide several quantitative parameters such as resting motor threshold (rMT), amplitude, volume of the MEP output curve, intracortical inhibition (ICI) and intracortical facilitation (ICF). Previous studies revealed that a high rMT could be associated with poor motor functional outcomes [15]. Several studies showed that the amplitude or amplitude ratio could be used for predicting motor functional outcomes $[9,10]$. The MEP amplitude has been used as a neurophysiological measure of corticomotor excitability [16]. The MEP output curve refers to the increase in MEP amplitude with increasing stimulus intensity of MEP. It can assess neurons that are intrinsically less excitable or spatially further from the center of activation by TMS [17]. As compared with many studies indicating a correlation between functional outcome and responsiveness or rMT of MEP, there have been few studies investigating other quantitative parameters of MEP as predictors of motor functional outcome and their results were incongruent. Therefore, the objective of this study was to reveal the usability of TMS-induced MEP in the early subacute phase to predict motor functional outcomes. For this purpose, we analyzed the relationships between the results of TMS-induced MEP in the early subacute phase and motor function at 3 months in stroke patients.

\section{MATERIALS AND METHODS}

\section{Subjects}

This study was a retrospective study performed using medical records of patients who suffered a stroke and were admitted to Samsung Medical Center. Inclusion criteria were patients who had (1) unilateral stroke lesions, (2) undergone motor functional evaluation at 3 months after stroke, (3) been transferred to the department of rehabilitation within 3 weeks after stroke onset, and (4) received TMS-induced MEP to evaluate cortical excitability within 3 weeks after stroke onset. In the present study, the 'early subacute phase' is defined as from 1 week to 3 weeks after stroke onset.

The exclusion criteria were patients who (1) suffered recurrent stroke, (2) were diagnosed with bilateral lesions by MRI or CT, (3) had other major neurological disease which could affect the patients' function. A total of 113 patients (61 men, 52 women; mean age $57.8 \pm 12.2$ years) who suffered a first-ever stroke were included in the study.

\section{TMS-induced MEP}

For performing single-pulse TMS-induced MEP, the patients were seated in a reclining armchair with both hands pronated. Electromyography (EMG) data were recorded from the contralateral first dorsal interosseous muscle via surface electrodes. EMG activity was amplified using the Medelec Synergy EMG/EP system (Medelec, Oxford, UK), and the data were bandpass filtered at 10-2,000 kHz. Using a TMS system (Magstim BiStim2; Magstim Ltd., Carmarthenshire, UK) and a 70-mm figureof-eight coil, the optimal scalp location was determined. The electromagnetic current would flow perpendicular to the central sulcus because the handle of the coil was oriented $45^{\circ}$ posterior to the midline $[18,19]$. Next, a single-pulse TMS was repeatedly applied to that location to determine each patient's rMT, defined as the lowest intensity of stimulus necessary to produce a MEP peak-topeak amplitude $\geq 50 \mu \mathrm{V}$ in five out of the 10 consecutive trials. The rMT provides information about a central core of neurons in the muscle representation in the motor 
cortex and it likely reflects neuronal membrane excitability [20]. To verify whether the patient was relaxed prior to stimulation, the examiner monitored muscle activity by real-time EMG. Absent MEP was defined when it failed to appear after 3 successive discharges with maximal output.

We also evaluated the amplitude of MEP at $100 \%, 110 \%$, $120 \%, 130 \%, 140 \%$, and $150 \%$ of the rMT, latency of MEP, volume of the MEP output curve, and recruitment ratios by applying various stimulation intensities, respectively. For each intensity of the rMT, five sweeps of the MEP were collected, and the mean amplitude of the MEP was calculated [21]. In all patients, each single-pulse TMSinduced MEP was performed in both the affected and unaffected hemispheres using the same protocol.

For paired-pulse TMS-induced MEP, MEP amplitude could be changed depending on the interval between conditioning stimulus and test stimulus. In the present study, paired stimulations were delivered at $2 \mathrm{~ms}$ and $4 \mathrm{~ms}$ intervals for triggering ICI and at $10 \mathrm{~ms}$ and $15 \mathrm{~ms}$ intervals for triggering intracortical facilitation (ICF), respectively. Intensities of conditioning and test stimulus were $80 \%$ and $120 \%$ of the rMT. Reductions or increases in the test stimulus by conditioning pulse were expressed as a percentage of the unconditioned MEP amplitude [22].

\section{Motor function assessment}

Primary outcome of this study was each patient's motor function, which was measured by Fugl-Meyer Assessment scale (FMA). The FMA used in our study consisted of an upper extremity score (FMA-UL), a lower extremity score (FMA-LL), and the total score (FMA-T; the sum of both the upper and lower extremity scores). We assessed each participant's motor function in the early subacute phase and at 3 months after stroke.

\section{Statistical analysis}

Data analysis was carried out using IBM SPSS ver. 22 software (IBM, Armonk, NY, USA). We classified stroke patients into the MEP response group and the MEP nonresponse group according to the presence of MEP in the affected hemisphere. In the MEP response group, we also calculated the ratio of parameters of TMS-induced MEP between both sides to evaluate the imbalance of cortical excitability. We defined the ratio of each parameter by calculating the difference between both sides (value of the affected side/value of the unaffected side). The Shapiro-Wilk test was used to determine the distributional normality of all continuous variables (all variables were found to be normally distributed; $\mathrm{p}<0.05$ ). Independent t-tests and chi-square tests were used to compare the parameters between the MEP response and MEP nonresponse groups. In the MEP response group, correlation analysis was used to assess the correlation between motor function and MEP parameters. After correlation analysis, multiple regression analysis with significant variables was used to identify the meaningful independent prognostic factor. The $\mathrm{p}$-values less than 0.05 were considered statistically significant.

\section{RESULTS}

The clinical characteristics of patients are shown in Table 1. Of the 113 patients, 84 patients suffered an ischemic stroke and the other patients suffered a hemorrhagic stroke. With respect to the lesion location of stroke, 21, 75, and 17 patients were diagnosed as having cortical, subcortical, and brainstem lesions, respectively. After performing TMS-induced MEP, 40 patients and 73 patients were included in the MEP response group and the MEP non-response group, respectively. Comparison according to the responsiveness of MEP revealed that the FMA-T and FMA-UL were significantly higher in the MEP response group than in the MEP non-response group in both the early subacute phase and at 3 months after stroke onset $(\mathrm{p}<0.05)$ (Table 2).

In the MEP response group, the mean MEP amplitude at $120 \%$ of the rMT was $411.9 \pm 376.5 \mu \mathrm{V}$ and the mean rMT was $50.6 \pm 16.7$ (Table 1). For the correlation analysis between MEP parameters and FMA score, in the affected hemisphere, the rMT showed negative relationships with FMA-T (Pearson coefficient $=-0.437, \mathrm{p}=0.005$ ) and FMAUL (Pearson coefficient $=-0.418, p=0.007$ ). The rMT ratio also showed a negative relationship with FMA-T (Pearson coefficient $=-0.621, \mathrm{p}<0.001$ ) and FMA-UL (Pearson coefficient $=-0.623, p<0.001)$. Other MEP parameters such as MEP amplitude at any intensity, volume of the MEP output curve, recruitment ratio, latency, ICI or ICF showed no significant relationship with motor function at 3 months after stroke. In addition, each FMA score in the early subacute phase after stroke onset showed a positive relationship with each FMA score at 3 months after 
Table 1. Clinical characteristics, cortical excitability measurements, and functional assessments of patients with stroke

\begin{tabular}{lc}
\hline \multicolumn{1}{c}{ Parameter } & Value \\
\hline Age (yr) & $57.8 \pm 12.2(28-80)$ \\
Sex & \\
$\quad$ Male & 61 \\
$\quad$ Female & 52 \\
Stroke type & \\
$\quad$ Ischemic & 84 \\
\hline Hemorrhagic & 29 \\
Stroke lesion & \\
$\quad$ Cortical & 21 \\
\hline Subcortical & 75 \\
\hline Brainstem & 17 \\
Duration after stroke to \\
initial MEP (day)
\end{tabular}

Values are presented as mean \pm standard deviation (range) or number of patients.

MEP, motor evoked potential; TMS, transcranial magnetic stimulation; FMA, Fugl-Meyer Assessment scale; FMA-T, total scores of FMA; FMA-UL, upper limb scores of FMA; FMA-LL, lower limb scores of FMA.

stroke, respectively (Table 3 ).

In the multiple regression analysis, FMA score and rMT ratio, but not rMT in the early subacute phase were found to be independent prognostic factors for FMA$\mathrm{T}\left(\mathrm{R}^{2}=0.633, \mathrm{p}<0.001, \mathrm{~F}=0.011\right)$ and FMA-UL $\left(\mathrm{R}^{2}=0.604\right.$, $\mathrm{p}<0.001, \mathrm{~F}=0.012$ ) at 3 months after stroke (Table 4).

In the analysis according to the stroke type, the ischemic stroke group showed similar results. For the correlation analysis, the rMT in the affected hemisphere showed negative relationships with FMA-T (Pearson coefficient $=-0.401, \mathrm{p}=0.02$ ) and FMA-UL (Pearson coefficient $=-0.371, p=0.04)$. The rMT ratio also showed a negative relationship with FMA-T (Pearson coefficient $=-0.628$,
Table 2. Comparison between the MEP response group and the MEP non-response group

\begin{tabular}{|c|c|c|}
\hline & $\begin{array}{c}\text { MEP } \\
\text { response } \\
\text { group } \\
(n=40)\end{array}$ & $\begin{array}{c}\text { MEP } \\
\text { non-response } \\
\text { group } \\
(n=73)\end{array}$ \\
\hline \multicolumn{3}{|l|}{ FMA-T } \\
\hline Early subacute phase & $55.4 \pm 26.8^{*}$ & $28.5 \pm 21.4$ \\
\hline At 3 months after stroke & $78.4 \pm 22.3^{*}$ & $49.4 \pm 30.0$ \\
\hline \multicolumn{3}{|l|}{ FMA-UL } \\
\hline Early subacute phase & $36.9 \pm 19.0^{*}$ & $14.0 \pm 12.9$ \\
\hline At 3 months after stroke & $52.1 \pm 14.8^{*}$ & $27.9 \pm 21.0$ \\
\hline \multicolumn{3}{|l|}{ FMA-LL } \\
\hline Early subacute phase & $18.5 \pm 9.1^{*}$ & $14.5 \pm 10.5$ \\
\hline At 3 months after stroke & $26.3 \pm 8.4^{*}$ & $21.5 \pm 10.4$ \\
\hline \multicolumn{3}{|l|}{ Lesion site } \\
\hline Cortex & $9(22)$ & $12(16)$ \\
\hline Subcortex & $25(63)$ & $50(68)$ \\
\hline Brainstem & $6(15)$ & $11(15)$ \\
\hline \multicolumn{3}{|l|}{ Stroke type } \\
\hline Infarction & $31(77)$ & $53(73)$ \\
\hline Hemorrhage & $9(23)$ & $20(27)$ \\
\hline \multicolumn{3}{|c|}{$\begin{array}{l}\text { Values are presented as mean } \pm \text { standard deviation or } \\
\text { number }(\%) \text {. } \\
\text { MEP, motor evoked potential; FMA, Fugl-Meyer Assess- } \\
\text { ment scale; FMA-T, total scores of FMA; FMA-UL, upper } \\
\text { limb scores of FMA; FMA-LL, lower limb scores of FMA. } \\
\text { *p<0.05 compared with the MEP non-response group C. }\end{array}$} \\
\hline
\end{tabular}

$\mathrm{p}<0.001$ ) and FMA-UL (Pearson coefficient $=-0.629$, $\mathrm{p}<0.001$ ) (Table 5). However, multiple regression analysis revealed that the initial FMA score and rMT ratio, but not rMT were independent prognostic factors for FMA-T $\left(\mathrm{R}^{2}=0.717, \mathrm{p}<0.001, \mathrm{~F}=0.040\right)$ at 3 months after stroke. On the other hand, the hemorrhagic stroke group showed no relationship between MEP parameters and motor functional outcome. With respect to the stroke lesion classified as cortical, subcortical and brainstem, the result also showed no significant difference between each lesion site.

\section{DISCUSSION}

In the current study, we investigated the potential of TMS-induced MEP in the early subacute phase to predict motor function at 3 months after stroke onset. We could demonstrate two findings: (1) the MEP responsiveness 
Table 3. Correlations between MEP and functional assessments at 3 months after stroke

\begin{tabular}{|c|c|c|c|}
\hline & FMA-T & FMA-UL & FMA-LL \\
\hline \multicolumn{4}{|l|}{ Affected hemisphere } \\
\hline Resting motor threshold (\%) & $-0.437(0.005)^{* *}$ & $-0.418(0.007)^{* *}$ & $-0.421(0.007) * *$ \\
\hline \multicolumn{4}{|l|}{ Amplitude $(\mu \mathrm{V})$} \\
\hline At $100 \%$ of the rMT & $-0.046(0.77)$ & $-0.031(0.85)$ & $-0.068(0.67)$ \\
\hline At $110 \%$ of the rMT & $0.251(0.17)$ & $0.284(0.12)$ & $0.148(0.43)$ \\
\hline At $120 \%$ of the rMT & $0.217(0.22)$ & $0.275(0.12)$ & $0.084(0.64)$ \\
\hline At $130 \%$ of the rMT & $0.317(0.11)$ & $0.360(0.07)$ & $0.161(0.43)$ \\
\hline At $140 \%$ of the rMT & $0.292(0.17)$ & $0.335(0.11)$ & $0.108(0.62)$ \\
\hline At $150 \%$ of the rMT & $0.168(0.48)$ & $0.283(0.23)$ & $0.091(0.70)$ \\
\hline Volume of the MEP output curve & $-0.196(0.25)$ & $-0.241(0.16)$ & $-0.076(0.66)$ \\
\hline Recruitment ratio & $0.226(0.18)$ & $0.233(0.17)$ & $0.192(0.26)$ \\
\hline Latency of MEP (ms) & $0.067(0.68)$ & $0.056(0.73)$ & $0.077(0.63)$ \\
\hline ICI $2 \mathrm{~ms}(\%)$ & $0.141(0.58)$ & $0.088(0.73)$ & $0.178(0.48)$ \\
\hline ICI 4 ms (\%) & $0.094(0.71)$ & $0.034(0.90)$ & $0.154(0.54)$ \\
\hline ICF $10 \mathrm{~ms}(\%)$ & $0.164(0.52)$ & $0.041(0.87)$ & $0.294(0.24)$ \\
\hline ICF 15 ms (\%) & $0.243(0.33)$ & $0.201(0.42)$ & $0.238(0.34)$ \\
\hline \multicolumn{4}{|l|}{ Unaffected hemisphere } \\
\hline Resting motor threshold (\%) & $0.149(0.35)$ & $0.171(0.29)$ & $0.093(0.57)$ \\
\hline \multicolumn{4}{|l|}{ Amplitude $(\mu \mathrm{V})$} \\
\hline At $100 \%$ of the rMT & $-0.189(0.24)$ & $-0.213(0.18)$ & $-0.125(0.44)$ \\
\hline At $110 \%$ of the rMT & $-0.198(0.25)$ & $-0.246(0.16)$ & $-0.085(0.63)$ \\
\hline At $120 \%$ of the rMT & $-0.053(0.74)$ & $-0.091(0.57)$ & $0.020(0.90)$ \\
\hline At $130 \%$ of the rMT & $-0.181(0.31)$ & $-0.204(0.25)$ & $-0.111(0.53)$ \\
\hline At $140 \%$ of the rMT & $-0.300(0.09)$ & $-0.328(0.06)$ & $-0.202(0.25)$ \\
\hline At $150 \%$ of the rMT & $-0.257(0.16)$ & $-0.269(0.14)$ & $-0.195(0.29)$ \\
\hline Volume of the MEP output curve & $0.249(0.08)$ & $0.215(0.14)$ & $0.280(0.05)$ \\
\hline Recruitment ratio & $-0.001(0.99)$ & $-0.013(0.94)$ & $0.022(0.90)$ \\
\hline Latency of MEP (ms) & $0.096(0.55)$ & $0.081(0.61)$ & $0.113(0.48)$ \\
\hline ICI $2 \mathrm{~ms}(\%)$ & $0.146(0.47)$ & $0.108(0.59)$ & $0.200(0.32)$ \\
\hline ICI $4 \mathrm{~ms}(\%)$ & $0.165(0.41)$ & $0.131(0.52)$ & $0.210(0.29)$ \\
\hline ICF $10 \mathrm{~ms}(\%)$ & $-0.094(0.64)$ & $-0.085(0.68)$ & $-0.102(0.61)$ \\
\hline ICF 15 ms (\%) & $-0.028(0.89)$ & $-0.051(0.80)$ & $0.013(0.95)$ \\
\hline \multicolumn{4}{|l|}{ Ratio } \\
\hline Resting motor threshold & $-0.621(<0.001)^{* *}$ & $-0.623(<0.001)^{* *}$ & $-0.546(<0.001) * *$ \\
\hline Amplitude at $120 \%$ of the rMT & $0.161(0.27)$ & $0.141(0.33)$ & $0.023(0.90)$ \\
\hline Volume of the MEP output curve & $-0.245(0.15)$ & $-0.309(0.07)$ & $-0.084(0.63)$ \\
\hline Latency of MEP & $-0.048(0.77)$ & $-0.045(0.78)$ & $-0.048(0.77)$ \\
\hline \multicolumn{4}{|l|}{ FMA in the early subacute phase } \\
\hline FMA-T & $0.750(<0.001)^{* *}$ & $0.760(<0.001)^{* *}$ & $0.647(<0.001)^{* *}$ \\
\hline FMA-UL & $0.704(<0.001)^{* *}$ & $0.727(<0.001)^{* *}$ & $0.584(<0.001)^{* *}$ \\
\hline FMA-LL & $0.737(<0.001)^{* *}$ & $0.718(<0.001)^{* *}$ & $0.686(<0.001)^{* *}$ \\
\hline
\end{tabular}

Values are presented as correlation coefficients ( $\mathrm{p}$-values).

FMA, Fugl-Meyer Assessment scale; FMA-T, total scores of FMA; FMA-UL, upper limb scores of FMA; FMA-LL, lower limb scores of FMA; MEP, motor evoked potential; rMT, resting motor threshold; ICI, intracortical inhibition; ICF, intracortical facilitation.

${ }^{* *} \mathrm{p}<0.01$. 
Table 4. Stepwise multiple regression analyses

\begin{tabular}{|c|c|c|c|c|c|}
\hline Independent variable & $\mathbf{R}$ & $\mathbf{R}^{2}$ & ${\text { Adjusted } \mathrm{R}^{2}}^{2}$ & p-value & F-value \\
\hline \multicolumn{6}{|l|}{ FMA-T at 3 months after stroke } \\
\hline Step 1: FMA-T in the early subacute phase & 0.750 & 0.563 & 0.551 & $<0.001$ & 0.000 \\
\hline Step 2: FMA-T, rMT ratio in the early subacute phase & 0.796 & 0.633 & 0.613 & $<0.001$ & 0.011 \\
\hline \multicolumn{6}{|l|}{ FMA-UL at 3 months after stroke } \\
\hline Step 1: FMA-UL in the early subacute phase & 0.727 & 0.529 & 0.517 & $<0.001$ & 0.000 \\
\hline Step 2: FMA-UL, rMT ratio in the early subacute phase & 0.777 & 0.604 & 0.583 & $<0.001$ & 0.012 \\
\hline
\end{tabular}

FMA, Fugl-Meyer Assessment scale; FMA-T, total scores of FMA; FMA-UL, upper limb scores of FMA; FMA-LL, lower limb scores of FMA; rMT, resting motor threshold.

was one of the strong tools to predict motor function at 3 months after stroke, (2) the rMT ratio in the early subacute phase was a significant independent prognostic factor for motor function at 3 months after stroke.

The MEP responsiveness as a parameter for predicting motor outcome was comparable with the results of previous studies [7-12]. MEP responsiveness showed high positive predictability of motor functional recovery compared to diffusion tensor tractography which showed high negative predictability [6]. Song et al. [12] reported that the low limit value of fractional anisotropy in the cerebral peduncle on the affected side was correlated with the poor hemiplegic limb motor function recovery in MEP non-responsive stroke patients because Wallerian degeneration of the cerebral peduncle occurred in accordance with changes in motor evoked potentials. However, there is no specific clue to reveal how MEP measures can be used as a predictive tool for motor recovery in MEPresponsive patients.

The results of this study revealed that the rMT and rMT ratio of MEP are significant tools for predicting motor functional outcome in MEP-responsive stroke patients. Furthermore, the multiple regression analysis revealed that rMT ratio in the early subacute phase was an independent prognostic factor for motor functional recovery. On the other hand, it showed no significant differences depending on the stroke type and lesion location, which could have been caused by the small number of patients. Motor function in the subacute phase is the strongest predictor for motor functional outcome in stroke patients [8]. rMT means threshold of the pyramidal tract responding to the magnetic stimulus which can reflect neuronal membrane excitability, thus a higher rMT ratio can be interpreted as an imbalance of motor threshold between bilateral primary motor cortices $[23,24]$. In addition,
Takechi et al. [25] showed that higher rMT may predict poor clinical recovery. In this context, this result suggests that the imbalance of motor threshold between bilateral primary motor cortices can be an important factor associated with late motor recovery. In stroke patients, neuronal excitability decreases, which leads to higher motor threshold in the affected side causing an imbalance of motor threshold $[20,26]$. Furthermore, several studies postulated that the interhemispheric balance is disrupted in stroke patients and this caused suppression of ipsilesional cortical excitability by the contralesional hemisphere [27-29]. Prashantha et al. [26] described that disruption of the transcallosal interaction could be the possible mechanism causing an imbalance of rMT. Considering these findings together, we can postulate that the patients with a higher rMT ratio may present lower cortical responsibility in the lesioned hemisphere, indicating lower cortical excitability. Therefore, it can be inferred that interhemispheric inhibition could be the possible mechanism of imbalance of bilateral hemispheres, interfering with motor recovery in stroke patients. In other words, the contralesional hemisphere may provide more suppression via the transcallosal inhibitory circuit, and consequently inhibit the recovery of ipsilesional motor cortical function.

With respect to the other MEP parameters such as amplitude or latency as prediction tools for motor recovery, several studies reported on the amplitude or amplitude ratio of MEP and they showed incongruent results $[9,10]$. Choi et al. [9] indicated that MEP responsiveness and amplitude ratio are significantly associated with the upper extremity function evaluated by Brunnstrom stage of hand recovery and the Modified Barthel Index at the time of admission. However, in this study, we could not reproduce the relationship between amplitude or ampli- 
Table 5. Correlations between MEP and functional assessments at 3 months after ischemic stroke

\begin{tabular}{|c|c|c|c|}
\hline & FMA-T & FMA-UL & FMA-LL \\
\hline \multicolumn{4}{|l|}{ Affected hemisphere } \\
\hline Resting motor threshold (\%) & $-0.401(0.02)^{*}$ & $-0.371(0.04)^{*}$ & $-0.405(0.02)^{*}$ \\
\hline \multicolumn{4}{|l|}{ Amplitude $(\mu \mathrm{V})$} \\
\hline At $100 \%$ of the rMT & $-0.025(0.89)$ & $-0.018(0.92)$ & $-0.036(0.85)$ \\
\hline At $110 \%$ of the rMT & $0.262(0.24)$ & $0.293(0.19)$ & $0.161(0.48)$ \\
\hline At $120 \%$ of the rMT & $0.130(0.53)$ & $0.190(0.35)$ & $0.003(0.99)$ \\
\hline At $130 \%$ of the rMT & $0.197(0.43)$ & $0.261(0.30)$ & $0.023(0.93)$ \\
\hline At $140 \%$ of the rMT & $0.098(0.72)$ & $0.178(0.51)$ & $-0.087(0.75)$ \\
\hline At $150 \%$ of the rMT & $0.007(0.98)$ & $0.174(0.55)$ & $-0.273(0.35)$ \\
\hline Volume of the MEP output curve & $0.424(0.17)$ & $0.032(0.91)$ & $0.346(0.16)$ \\
\hline Recruitment ratio & $0.150(0.41)$ & $0.132(0.47)$ & $0.164(0.37)$ \\
\hline Latency of MEP (ms) & $-0.008(0.98)$ & $0.009(0.97)$ & $-0.034(0.91)$ \\
\hline ICI $2 \mathrm{~ms}(\%)$ & $0.058(0.84)$ & $0.032(0.91)$ & $0.080(0.79)$ \\
\hline ICI $4 \mathrm{~ms}(\%)$ & $-0.009(0.98)$ & $-0.117(0.69)$ & $0.166(0.57)$ \\
\hline ICF $10 \mathrm{~ms}(\%)$ & $0.404(0.15)$ & $0.322(0.26)$ & $0.396(0.16)$ \\
\hline ICF $15 \mathrm{~ms}(\%)$ & $0.424(0.17)$ & $0.032(0.91)$ & $0.346(0.16)$ \\
\hline \multicolumn{4}{|l|}{ Unaffected hemisphere } \\
\hline Resting motor threshold (\%) & $0.240(0.19)$ & $0.277(0.13)$ & $0.146(0.43)$ \\
\hline \multicolumn{4}{|l|}{ Amplitude $(\mu \mathrm{V})$} \\
\hline At $100 \%$ of the rMT & $-0.223(0.22)$ & $-0.253(0.16)$ & $-0.145(0.43)$ \\
\hline At $110 \%$ of the rMT & $-0.208(0.31)$ & $-0.252(0.21)$ & $-0.095(0.65)$ \\
\hline At $120 \%$ of the rMT & $-0.251(0.23)$ & $-0.267(0.20)$ & $-0.179(0.39)$ \\
\hline At $130 \%$ of the rMT & $-0.181(0.31)$ & $-0.204(0.25)$ & $-0.111(0.53)$ \\
\hline At $140 \%$ of the rMT & $-0.339(0.10)$ & $-0.360(0.08)$ & $-0.242(0.24)$ \\
\hline At $150 \%$ of the rMT & $-0.278(0.19)$ & $-0.283(0.18)$ & $-0.221(0.29)$ \\
\hline \multicolumn{4}{|l|}{ Volume of the MEP output curve } \\
\hline Recruitment ratio & $0.269(0.38)$ & $0.325(0.26)$ & $0.252(0.12)$ \\
\hline Latency of MEP (ms) & $0.154(0.40)$ & $0.137(0.46)$ & $0.167(0.36)$ \\
\hline ICI $2 \mathrm{~ms}(\%)$ & $0.126(0.58)$ & $0.095(0.67)$ & $0.170(0.45)$ \\
\hline ICI $4 \mathrm{~ms}(\%)$ & $0.117(0.60)$ & $0.088(0.70)$ & $0.159(0.48)$ \\
\hline ICF $10 \mathrm{~ms}(\%)$ & $-0.081(0.72)$ & $-0.071(0.75)$ & $-0.092(0.68)$ \\
\hline ICF $15 \mathrm{~ms}(\%)$ & $0.041(0.86)$ & $0.006(0.98)$ & $0.099(0.66)$ \\
\hline \multicolumn{4}{|l|}{ Ratio } \\
\hline Resting motor threshold & $-0.628(<0.001)^{* *}$ & $-0.629(<0.001)^{* *}$ & $-0.551(<0.001)^{* *}$ \\
\hline Amplitude at $120 \%$ of the rMT & $0.071(0.73)$ & $0.117(0.57)$ & $-0.021(0.92)$ \\
\hline Volume of the MEP output curve & $-0.267(0.17)$ & $-0.342(0.11)$ & $-0.108(0.86)$ \\
\hline Latency of MEP & $-0.024(0.90)$ & $-0.029(0.88)$ & $-0.012(0.95)$ \\
\hline \multicolumn{4}{|l|}{ FMA in the early subacute phase } \\
\hline FMA-T & $0.824(<0.001)^{* *}$ & $0.825(<0.001)^{* *}$ & $0.724(<0.001)^{* *}$ \\
\hline FMA-UL & $0.784(<0.001)^{* *}$ & $0.799(<0.001)^{* *}$ & $0.664(<0.001)^{* *}$ \\
\hline FMA-LL & $0.760(<0.001)^{* *}$ & $0.732(<0.001)^{* *}$ & $0.719(<0.001)^{* *}$ \\
\hline
\end{tabular}

Values are presented as correlation coefficients (p-values).

FMA, Fugl-Meyer Assessment scale; FMA-T, total scores of FMA; FMA-UL, upper limb scores of FMA; FMA-LL, lower limb scores of FMA; MEP, motor evoked potential; rMT, resting motor threshold; ICI, intracortical inhibition; ICF, intracortical facilitation.

${ }^{*} \mathrm{p}<0.05,{ }^{* *} \mathrm{p}<0.01$. 
tude ratio of MEP and motor functional outcome such as FMA scores. Kim et al. [10] also indicated that MEP responsiveness and amplitude ratio are significantly correlated with the MRC scale and the Modified Barthel Index. However, both these studies investigated the correlation between MEP parameters and motor function and both studies assessed patients in the subacute stage of stroke, which was different from the present study which investigated long-term functional outcomes. In addition, considering that the amplitude of MEP represents the corticospinal excitability of the M1 [23], it can be affected by temporal dispersion of corticospinal fiber tract conduction, which can confound the results [23]. In addition, the amplitude of MEP can be influenced by other contributing factors such as height or status of peripheral nerves and muscles [30]. Moreover, amplitude of MEP in the affected hemisphere may not represent the absolute value of cortical excitability. The latency of MEP might be influenced by peripheral nerve conduction time as well as central conduction time [23]. Because cortical motor output is the net result of the interplay between multiple systems that exert excitatory or inhibitory influences on the corticospinal neurons, ICI and ICF could represent intrinsic intracortical connectivity and cortical plasticity. ICI is suppressed on the affected side in the first few weeks after the ictus $[15,27,31,32]$, whereas ICF is consistently normal $[27,31,32]$. This suggests that the balance of excitability in intracortical circuits shifts towards excitation. Whether the reduction of ICI relates to the degree of disability is rather controversial [31,32]. Several studies showed that interventions such as motor task training, active or passive training, transcranial direct current stimulation could be related to reduced ICI, thus facilitating motor recovery [22,25,33-38]. However, the exact mechanism and causality of the correlation remain unclear and interpretation of ICI and ICF is complicated because they could be affected by various factors like position, intensity of test stimulus, and medication $[39,40]$. Taking all these aspects together, we can postulate that the rMT ratio may better reflect the changes in the motor cortical excitability after stroke than the other parameters of MEP.

This study has some limitations. First, this study retrospectively analyzes the patients who were transferred to our hospital and underwent inpatient rehabilitation therapy after stroke; therefore, there may be a bias of analyz- ing significantly affected patients. Second, the initial time period for obtaining first MEP data is rather variable, ranging from 1 to 3 weeks after stroke. Finally, the present study cannot identify whether stroke type or lesion location could affect the relationship between MEP parameters and motor functional outcomes. Clinical recovery after stroke depends on the ability to activate intrinsic cortical connections in the most efficient manner as well as the activation of corticospinal excitability itself. Some insight into the mechanisms of recovery in these individuals could be provided by more complex TMS measures that examine function of the intrinsic cortical pathways. Therefore, prospective investigations are required in future to overcome these limitations and to obtain a better predictive value of MEP parameters for motor recovery after stroke.

In addition to the responsiveness of MEP being an important factor for predicting motor function, the rMT ratio in the early subacute phase was also significantly correlated with motor recovery of the upper limb at 3 months after stroke onset. The quantitative measure of TMS-induced MEP could be used as a parameter to predict motor recovery in patients with stroke.

\section{CONFLICT OF INTEREST}

No potential conflict of interest relevant to this article was reported.

\section{ACKNOWLEDGMENTS}

This study was supported by a National Research Foundation of Korea (NRF) grant funded by the Korean government (MSIP) (NRF-2014R1A2A1A01005128).

\section{REFERENCES}

1. Minino AM, Murphy SL, Xu J, Kochanek KD. Deaths: final data for 2008. Natl Vital Stat Rep 2011;59:1-126.

2. Duncan PW, Goldstein LB, Matchar D, Divine GW, Feussner J. Measurement of motor recovery after stroke. Outcome assessment and sample size requirements. Stroke 1992;23:1084-9.

3. Groisser BN, Copen WA, Singhal AB, Hirai KK, Schaechter JD. Corticospinal tract diffusion abnormalities early after stroke predict motor outcome. 
Neurorehabil Neural Repair 2014;28:751-60.

4. Shelton FN, Reding MJ. Effect of lesion location on upper limb motor recovery after stroke. Stroke 2001; 32:107-12.

5. Nelles G, Spiekramann G, Jueptner M, Leonhardt G, Muller S, Gerhard H, et al. Evolution of functional reorganization in hemiplegic stroke: a serial positron emission tomographic activation study. Ann Neurol 1999;46:901-9.

6. Kwon YH, Son SM, Lee J, Bai DS, Jang SH. Combined study of transcranial magnetic stimulation and diffusion tensor tractography for prediction of motor outcome in patients with corona radiata infarct. J Rehabil Med 2011;43:430-4.

7. Rapisarda G, Bastings E, de Noordhout AM, Pennisi G, Delwaide PJ. Can motor recovery in stroke patients be predicted by early transcranial magnetic stimulation? Stroke 1996;27:2191-6.

8. Nascimbeni A, Gaffuri A, Imazio P. Motor evoked potentials: prognostic value in motor recovery after stroke. Funct Neurol 2006;21:199-203.

9. Choi TW, Jang SG, Yang SN, Pyun SB. Factors affecting the motor evoked potential responsiveness and parameters in patients with supratentorial stroke. Ann Rehabil Med 2014;38:19-28.

10. Kim GW, Won YH, Park SH, Seo JH, Ko MH. Can motor evoked potentials be an objective parameter to assess extremity function at the acute or subacute stroke stage? Ann Rehabil Med 2015;39:253-61.

11. Lee SY, Lim JY, Kang EK, Han MK, Bae HJ, Paik NJ. Prediction of good functional recovery after stroke based on combined motor and somatosensory evoked potential findings. J Rehabil Med 2010;42:16-20.

12. Song Z, Dang L, Zhou Y, Dong Y, Liang H, Zhu Z, et al. Why do stroke patients with negative motor evoked potential show poor limb motor function recovery? Neural Regen Res 2013;8:2713-24.

13. Hendricks HT, Pasman JW, van Limbeek J, Zwarts MJ. Motor evoked potentials in predicting recovery from upper extremity paralysis after acute stroke. Cerebrovasc Dis 2003;16:265-71.

14. Pennisi G, Rapisarda G, Bella R, Calabrese V, Maertens De Noordhout A, et al. Absence of response to early transcranial magnetic stimulation in ischemic stroke patients: prognostic value for hand motor recovery. Stroke 1999;30:2666-70.
15. Nardone R, Tezzon F. Inhibitory and excitatory circuits of cerebral cortex after ischaemic stroke: prognostic value of the transcranial magnetic stimulation. Electromyogr Clin Neurophysiol 2002;42:131-6.

16. Rossini PM, Burke D, Chen R, Cohen LG, Daskalakis Z, Di Iorio $\mathrm{R}$, et al. Non-invasive electrical and magnetic stimulation of the brain, spinal cord, roots and peripheral nerves: basic principles and procedures for routine clinical and research application. An updated report from an I.F.C.N. Committee. Clin Neurophysiol 2015;126:1071-107.

17. Hallett M, Chen R, Ziemann U, Cohen LG. Reorganization in motor cortex in amputees and in normal volunteers after ischemic limb deafferentation. Electroencephalogr Clin Neurophysiol Suppl 1999;51:1837.

18. Nitsche MA, Paulus W. Sustained excitability elevations induced by transcranial DC motor cortex stimulation in humans. Neurology 2001;57:1899-901.

19. Nitsche MA, Roth A, Kuo MF, Fischer AK, Liebetanz D, Lang N, et al. Timing-dependent modulation of associative plasticity by general network excitability in the human motor cortex. J Neurosci 2007;27:3807-12.

20.Ziemann U, Lönnecker S, Steinhoff BJ, Paulus W. Effects of antiepileptic drugs on motor cortex excitability in humans: a transcranial magnetic stimulation study. Ann Neurol 1996;40:367-78.

21. Uhm KE, Kim YH, Yoon KJ, Hwang JM, Chang WH. BDNF genotype influence the efficacy of rTMS in stroke patients. Neurosci Lett 2015;594:117-21.

22. Zhang X, Woolley DG, Swinnen SP, Feys H, Meesen R, Wenderoth N. Changes in corticomotor excitability and intracortical inhibition of the primary motor cortex forearm area induced by anodal tDCS. PLoS One 2014;9:e101496.

23. Groppa S, Oliviero A, Eisen A, Quartarone A, Cohen LG, Mall V, et al. A practical guide to diagnostic transcranial magnetic stimulation: report of an IFCN committee. Clin Neurophysiol 2012;123:858-82.

24. McConnell KA, Nahas Z, Shastri A, Lorberbaum JP, Kozel FA, Bohning DE, et al. The transcranial magnetic stimulation motor threshold depends on the distance from coil to underlying cortex: a replication in healthy adults comparing two methods of assessing the distance to cortex. Biol Psychiatry 2001;49:454-9.

25. Takechi U, Matsunaga K, Nakanishi R, Yamanaga $H$, 
Murayama N, Mafune K, et al. Longitudinal changes of motor cortical excitability and transcallosal inhibition after subcortical stroke. Clin Neurophysiol 2014;125:2055-69.

26. Prashantha DK, Sriranjini SJ, Sathyaprabha TN, Nagaraja D, Pal PK. Evaluation of the motor cortical excitability changes after ischemic stroke. Ann Indian Acad Neurol 2013;16:394-7.

27. Cicinelli P, Pasqualetti P, Zaccagnini M, Traversa R, Oliveri M, Rossini PM. Interhemispheric asymmetries of motor cortex excitability in the postacute stroke stage: a paired-pulse transcranial magnetic stimulation study. Stroke 2003;34:2653-8.

28. Takeuchi N, Oouchida Y, Izumi S. Motor control and neural plasticity through interhemispheric interactions. Neural Plast 2012;2012:823285.

29. Shimizu T, Hosaki A, Hino T, Sato M, Komori T, Hirai $\mathrm{S}$, et al. Motor cortical disinhibition in the unaffected hemisphere after unilateral cortical stroke. Brain 2002;125(Pt 8):1896-907.

30. Matamala JM, Nunez C, Lera L, Verdugo RJ, Sanchez $\mathrm{H}$, Albala C, et al. Motor evoked potentials by transcranial magnetic stimulation in healthy elderly people. Somatosens Mot Res 2013;30:201-5.

31. Liepert J, Hamzei F, Weiller C. Motor cortex disinhibition of the unaffected hemisphere after acute stroke. Muscle Nerve 2000;23:1761-3.

32. Manganotti P, Patuzzo S, Cortese F, Palermo A, Smania N, Fiaschi A. Motor disinhibition in affected and unaffected hemisphere in the early period of recovery after stroke. Clin Neurophysiol 2002;113:936-43.
33. Morishita T, Ninomiya M, Uehara K, Funase K. Increased excitability and reduced intracortical inhibition in the ipsilateral primary motor cortex during a fine-motor manipulation task. Brain Res 2011;1371: 65-73.

34. Blicher JU, Jakobsen J, Andersen G, Nielsen JF. Cortical excitability in chronic stroke and modulation by training: a TMS study. Neurorehabil Neural Repair 2009;23:486-93.

35. Garry MI, Kamen G, Nordstrom MA. Hemispheric differences in the relationship between corticomotor excitability changes following a fine-motor task and motor learning. J Neurophysiol 2004;91:1570-8.

36. Blicher JU, Nielsen JF. Cortical and spinal excitability changes after robotic gait training in healthy participants. Neurorehabil Neural Repair 2009;23:143-9.

37. Lotze M, Braun C, Birbaumer N, Anders S, Cohen LG. Motor learning elicited by voluntary drive. Brain 2003;126(Pt 4):866-72.

38. Takeuchi N, Tada T, Toshima M, Ikoma K. Correlation of motor function with transcallosal and intracortical inhibition after stroke. J Rehabil Med 2010;42:962-6.

39. Paulus W, Classen J, Cohen LG, Large CH, Di Lazzaro V, Nitsche M, et al. State of the art: Pharmacologic effects on cortical excitability measures tested by transcranial magnetic stimulation. Brain Stimul 2008; 1:151-63.

40. Kothari M, Svensson P, Nielsen JF, Baad-Hansen L. Influence of position and stimulation parameters on intracortical inhibition and facilitation in human tongue motor cortex. Brain Res 2014;1557:83-9. 